\title{
Angle dependence of Andreev scattering at semiconductor-superconductor interfaces
}

\author{
Mortensen, Asger; Flensberg, Karsten; Jauho, Antti-Pekka
}

Published in:

Physical Review B

Link to article, DOI:

10.1103/PhysRevB.59.10176

Publication date:

1999

Document Version

Publisher's PDF, also known as Version of record

Link back to DTU Orbit

Citation (APA):

Mortensen, A., Flensberg, K., \& Jauho, A-P. (1999). Angle dependence of Andreev scattering at semiconductorsuperconductor interfaces. Physical Review B, 59(15), 10176-10182.

https://doi.org/10.1103/PhysRevB.59.10176

\section{General rights}

Copyright and moral rights for the publications made accessible in the public portal are retained by the authors and/or other copyright owners and it is a condition of accessing publications that users recognise and abide by the legal requirements associated with these rights.

- Users may download and print one copy of any publication from the public portal for the purpose of private study or research.

- You may not further distribute the material or use it for any profit-making activity or commercial gain

- You may freely distribute the URL identifying the publication in the public portal

If you believe that this document breaches copyright please contact us providing details, and we will remove access to the work immediately and investigate your claim. 


\title{
Angle dependence of Andreev scattering at semiconductor-superconductor interfaces
}

\author{
Niels Asger Mortensen \\ Mikroelektronik Centret, Technical University of Denmark, Building 345 east, DK-2800 Lyngby, Denmark \\ and Department of Physics, Technical University of Denmark, Building 309, DK-2800 Lyngby, Denmark \\ Karsten Flensberg \\ Danish Institute of Fundamental Metrology, Building 307, Anker Engelunds Vej 1, DK-2800 Lyngby, Denmark \\ Antti-Pekka Jauho \\ Mikroelektronik Centret, Technical University of Denmark, Building 345 east, DK-2800 Lyngby, Denmark
}

(Received 6 July 1998; revised manuscript received 2 November 1998)

\begin{abstract}
We study the angle dependence of the Andreev scattering at a semiconductor-superconductor interface, generalizing the one-dimensional theory of Blonder, Tinkham, and Klapwijk (BTK). An increase of the momentum parallel to the interface leads to suppression of the probability of Andreev reflection and increase of the probability of normal reflection. We show that in the presence of a Fermi velocity mismatch between the semiconductor and the superconductor the angles of incidence and transmission are related according to the well-known Snell's law in optics. As a consequence there is a critical angle of incidence above which only normal reflection exists. For two- and three-dimensional interfaces a lower excess current compared to ballistic transport with perpendicular incidence is found. Thus, the one-dimensional BTK model overestimates the barrier strength for two- and three-dimensional interfaces. [S0163-1829(99)01615-X]
\end{abstract}

\section{INTRODUCTION}

An electronlike quasiparticle incident on a normal conductor-superconductor (NS) interface from the normal side may become Andreev reflected into a holelike quasiparticle with reversal of the signs of all three velocity components (retroflection) and of the energy (relative to the Fermi level) as shown by Andreev. ${ }^{1}$ Later, Blonder, Tinkham, and Klapwijk $^{2}$ (BTK) calculated the scattering probabilities at a NS interface within a model where the scattering at the interface was represented by a delta-function potential barrier. The calculations were based on the Bogoliubov-de Gennes (BdG) formalism, ${ }^{3}$ for a one-dimensional (1D) geometry thus ignoring all effects due to quasiparticles with a momentum parallel to the interface.

The BTK model has been widely used by experimentalists to model normal-metal-superconductor junctions, and it has despite of its inherent approximations been quite successful in describing the main features of these devices. The quality of the junction interface has conveniently been parametrized in terms of the normalized delta-function barrier strength.

A more complete theory was developed by Arnold ${ }^{4}$ using nonequilibrium Green's-function techniques. The theory by Arnold furthermore takes the three-dimensional (3D) nature of the interface into account. However, the resulting expressions are complicated and require substantial numerical work. Generalization of the BTK model to tunnel barriers other than delta-function scattering potentials has been done by Kupka in a number of papers. ${ }^{5,6}$ Recently, $\mathrm{Kupka}^{6}$ generalized the more realistic tunnel barrier model to include the angel dependence of the scattering. He found that by treating the scattering problem in the correct three-dimensional picture, the effective Andreev scattering is reduced and the normal scattering probability is enhanced. Chaudhuri and
Bagwell ${ }^{7}$ and De Raedt, Michielsen, and Klapwijk ${ }^{8}$ have also considered the angle dependence in their applications of the $\mathrm{BdG}$ formalism to the transport properties of NS interfaces. However, except for the 1D work of Blonder and Tinkham ${ }^{9}$ the above-mentioned papers all focused on the case where there is no mismatch between densities (and hence Fermi wavelengths) or between effective band masses of the two materials forming the NS junction. In the case of SNS junctions, Kupriyanov ${ }^{10}$ included effects of the parallel degree of freedom and different Fermi velocities of the $\mathrm{N}$ and $\mathrm{S}$ regions in his application of the Eilenberger equations to the $\mathrm{dc}$ Josephson current in junctions with clean interfaces. Using the BdG formalism, the effect of different Fermi velocities and effective masses was also considered by Schüssler and Kümmel $^{11}$ and Chrestin, Matsuyama, and Merkt ${ }^{12}$ in their numerical studies of the dc Josephson current in Nb-InAs- $\mathrm{Nb}$ junctions.

Since much of the development in the recent years has been in structures where superconductors are combined with semiconductors, the goal of this paper is an analytical study of the importance of the different quasiparticle propagation in the two materials, when the degrees of freedom parallel to the interface and effects of the unequal Fermi velocities and Fermi wavelengths are taken into account. This is motivated by the observation that Andreev scattering cannot occur above a critical angle where the momentum can no longer be conserved. The critical angle depends on the ratio of the carrier density of the semiconductor to the density of the superconductor. Therefore, one may expect larger differences between 1D and 2D or 3D junctions for the case of a finite Fermi wave-vector mismatch, which is indeed what we find.

The effect of the angle dependence of the Andreev scat- 
tering probability is however somewhat suppressed by the fact that the current is carried mostly by particles incoming perpendicular to the interface in $2 \mathrm{D}$ or at angle of $45^{\circ}$ in $3 \mathrm{D}$. Therefore, we suggest an experiment where the angle dependence of Andreev scattering is probed in a more direct fashion, namely a mesoscopic device which explores the ballistic motion of quasiparticles and where the angle of incidence can be varied. Such a device is possible due to the advances in fabrication of mesoscopic semiconductor-superconductor interfaces (see e.g., Ref. 13), which have made it possible to study Andreev scattering in the ballistic regime.

The paper is organized as follows: In Sec. II, the BdG formalism is introduced, and in Sec. III, the scattering probabilities at the interface are calculated. These scattering probabilities are used in Sec. IV to calculate current-voltage characteristics and related quantities. In Sec. V an experiment is suggested and, finally, in Sec. VI discussions and conclusions are given.

\section{THE BOGOLIUBOV-de GENNES FORMALISM}

The BdG equations

$$
\left(\begin{array}{cc}
\hat{\mathcal{H}}_{0}(\mathbf{r}) & \Delta(\mathbf{r}) \\
\Delta^{*}(\mathbf{r}) & -\hat{\mathcal{H}}_{0}^{*}(\mathbf{r})
\end{array}\right) \psi(\mathbf{r})=E \psi(\mathbf{r})
$$

provide a microscopic formalism for studying inhomogeneous superconductors and NS interfaces. ${ }^{3}$ Here, $\Delta(\mathbf{r})$ is the pairing potential and $\hat{\mathcal{H}}_{0}(\mathbf{r})$ is the Hamiltonian. In a general nonequilibrium situation, the Hamiltonian includes either a time-dependent vector potential or a spatially dependent scalar potential. However, we follow BTK (Ref. 2) and neglect the effect of a finite bias on the scattering probabilities, which is justified if the height of the tunnel barrier is much higher than the applied voltage and/or energy of the carriers (relative to the Fermi level). ${ }^{6}$ For an interface where the position of the conduction band and the effective mass change across the interface, we use the effective mass approximation $^{14,15}$

$$
\hat{\mathcal{H}}_{0}(\mathbf{r})=-\hat{\nabla} \frac{\hbar^{2}}{2 m^{\star}(\mathbf{r})} \hat{\nabla}+U(\mathbf{r})-\mu,
$$

where $U(\mathbf{r})$ is total electrostatic potential, and $\mu$ is the chemical potential. This approximation describes the spatial dependence of the dispersion relation, and the form of the Hamiltonian ensures conservation of the probability current. For a discussion of justifications of this approach, see Refs. 14 and 15 and references therein. We assume a parabolic dispersion so that the effective mass $m^{\star}$ does not depend on energy (or momentum).

The solutions to Eq. (1) are vectors in the so-called electron-hole space (Nambu space), $\psi(\mathbf{r})=[u(\mathbf{r}), v(\mathbf{r})]^{T}$, where $u(\mathbf{r})$ is the electronlike quasiparticle amplitude satisfying an ordinary electronlike Schrödinger equation and $v(\mathbf{r})$ is the holelike quasiparticle amplitude satisfying a timereversed Schrödinger equation. In electron-hole space, a probability current density can be associated with the wave function, and is given by $2,3,14$

$$
\mathbf{J}_{p}=\hbar \operatorname{Im}\left\{u^{*}(\mathbf{r}) \frac{1}{m^{\star}(\mathbf{r})} \hat{\nabla} u(\mathbf{r})-v^{*}(\mathbf{r}) \frac{1}{m^{\star}(\mathbf{r})} \hat{\nabla} v(\mathbf{r})\right\}
$$

The BdG equations and the conservation of the probability current density form the basis for our treatment of scattering of quasiparticles at the NS interface. Equation (1) is used in calculating scattering amplitudes and the corresponding scattering probabilities are found using Eq. (3).

\section{SCATTERING OF QUASIPARTICLES AT A NS INTERFACE}

We consider a planar NS interface lying in the $x y$-plane at $z=0$ with a semi-infinite nonsuperconducting material for $z$ $<0$ and a semi-infinite superconductor for $z>0$. The superconducting order parameter is assumed to vary in space only along the $z$ direction. In order to solve the $\mathrm{BdG}$ equations, we include only scattering at the NS interface. Following BTK, we model the scattering at the interface by a delta-function potential

$$
U(\mathbf{r})=H \delta(z),
$$

where $H$ is the strength of the potential barrier. For simplicity, we neglect the phase of the pairing potential since only the absolute value is important for the considered geometry. Furthermore, to avoid self-consistent calculations, we take the pairing potential to be zero in the normal conductor and uniform in the superconductor, i.e.,

$$
\Delta(\mathbf{r})=\Delta_{0} \Theta(z)
$$

where $\Delta_{0}$ is the BCS value of the energy gap and $\Theta(z)$ is a Heaviside function. Similarly for the effective masses of the two materials, we assume that the mass changes abruptly across the interface

$$
m^{\star}(\mathbf{r})=m^{(\mathrm{N})} \Theta(-z)+m^{(\mathrm{S})} \Theta(z),
$$

where $m^{(\mathrm{N})}$ and $m^{(\mathrm{S})}$ are the effective masses of the normal conductor and the superconductor, respectively. Equations (4)-(6) represent the simplest forms of $U(\mathbf{r}), \Delta(\mathbf{r})$, and $m^{\star}(\mathbf{r})$ still capturing the main physics of the NS interface.

Due to the simple form of the NS barrier potential, the pairing potential and the effective mass, we can separate the variables and express the solutions in the parallel direction as plane waves, i.e., $\psi^{(\mathrm{N}, \mathrm{S})}(\mathbf{r})=\exp \left[i\left(k_{x}^{(\mathrm{N}, \mathrm{S})} x+k_{y}^{(\mathrm{N}, \mathrm{S})} y\right)\right] \psi^{(\mathrm{N}, \mathrm{S})}$ $\times(z)$, where the superscript $(\mathrm{N}, \mathrm{S})$ refers to the nonsuperconducting or superconducting sides, respectively. Substituting this Ansatz into Eq. (1), yields the effective BdG equations for the $z$ direction 


$$
\left(\begin{array}{cc}
{\left[\begin{array}{c}
\hbar^{2} \\
\left.2 m^{(\mathrm{N}, \mathrm{S})} \frac{\partial^{2}}{\partial z^{2}}+H \delta(z)-\mu_{\mathrm{eff}}^{(\mathrm{N}, \mathrm{S})}\right]
\end{array}\right.} & \Delta_{0} \Theta(z) \\
\Delta_{0} \Theta(z) & \left.-\left[-\frac{\hbar^{2}}{2 m^{(\mathrm{N}, \mathrm{S})}} \frac{\partial^{2}}{\partial z^{2}}+H \delta(z)-\mu_{\mathrm{eff}}^{(\mathrm{N}, \mathrm{S})}\right]\right) \psi(z)=E \psi(z),
\end{array}\right.
$$

where the effective chemical potential is defined as

$$
\mu_{\mathrm{eff}}^{(\mathrm{N}, \mathrm{S})} \equiv \mu^{(\mathrm{N}, \mathrm{S})}-\frac{\hbar^{2}}{2 m^{(\mathrm{N}, \mathrm{S})}}\left(\left[k_{x}^{(\mathrm{N}, \mathrm{S})}\right]^{2}+\left[k_{y}^{(\mathrm{N}, \mathrm{S})}\right]^{2}\right) .
$$

Equation (7) is mathematically identical to the $1 \mathrm{D} \mathrm{BdG}$ equations considered by BTK, and, therefore, we expect similar results for the scattering probabilities. We adopt the notation of BTK (Ref. 2) and all formulas for the eigenstates, scattering states, wave vectors $\left(q^{ \pm}\right.$and $\left.k^{ \pm}\right)$etc. are equivalent to those of BTK, but with the important difference that the chemical potential is replaced by an effective chemical potential, which depends on the parallel momentum according to Eq. (8).

We follow BTK and consider an electronlike quasiparticle incident on the NS interface from the normal side. At the interface it has an amplitude $a$ of undergoing Andreev reflection, $b$ of normal reflection, $c$ of normal transmission, and $d$ of Andreev transmission. The scattering amplitudes are obtained by matching the scattering states at the NS interface, using the appropriate boundary conditions for a deltafunction potential barrier (see, e.g. Refs. 14 and 16). The matching results in following linear system determining $a, b, c$, and $d$

$$
\left(\begin{array}{cccc}
0 & 1 & -u_{0} & -v_{0} \\
1 & 0 & -v_{0} & -u_{0} \\
0 & \frac{2 H}{\hbar^{2}}-i \frac{q^{+}}{m^{(\mathrm{N})}} & -i \frac{k^{+}}{m^{(\mathrm{S})}} u_{0} & i \frac{k^{-}}{m^{(\mathrm{S})}} v_{0} \\
\frac{2 H}{\hbar^{2}}+i \frac{q^{-}}{m^{(\mathrm{N})}} & 0 & -i \frac{k^{+}}{m^{(\mathrm{S})}} v_{0} & i \frac{k^{-}}{m^{(\mathrm{S})}} u_{0}
\end{array}\right)\left(\begin{array}{l}
a \\
b \\
c \\
d
\end{array}\right)=\left(\begin{array}{c}
-1 \\
0 \\
-\frac{2 H}{\hbar^{2}}-i \frac{q^{+}}{m^{(\mathrm{N})}} \\
0
\end{array}\right)
$$

Though complicated, the exact scattering probabilities can now be found and numerical results for different values of the effective chemical potential have been given by $\breve{S i p r}$ and Györffy. ${ }^{17}$ However, the calculations may be simplified significantly for materials with a high Fermi energy compared to the temperatures or bias voltages of interest. In this limit transport only takes place near the Fermi level.

We choose polar coordinates and allow for a Fermi velocity mismatch by considering a wave vector on the normal side given by $\mathbf{k}^{(\mathrm{N})}=k_{\mathrm{F}}^{(\mathrm{N})}(\sin \theta \cos \phi, \sin \theta \sin \phi, \cos \theta)$ and a wave vector on the superconducting side with $\left|\mathbf{k}^{(\mathrm{S})}\right|=k_{\mathrm{F}}^{(\mathrm{S})}$.

The boundary conditions are satisfied only if $k_{x}^{(\mathrm{N})}=k_{x}^{(\mathrm{S})}$ and $k_{y}^{(\mathrm{N})}=k_{y}^{(\mathrm{S})}$, as dictated by the translational invariance along the interface. This means that $k_{z}^{(\mathrm{S})}$ $=k_{\mathrm{F}}^{(\mathrm{S})} \sqrt{1-r_{k}^{2} \sin ^{2} \theta}$, where the Fermi momentum ratio is given by $r_{k} \equiv k_{\mathrm{F}}^{(\mathrm{N})} / k_{\mathrm{F}}^{(\mathrm{S})}$. The wave vectors on the normal conducting side can now be written as $q^{ \pm}$ $=k_{\mathrm{F}}^{(\mathrm{N})} \sqrt{\cos ^{2} \theta \pm E / \mu^{(\mathrm{N})}}$ and on the superconducting side we

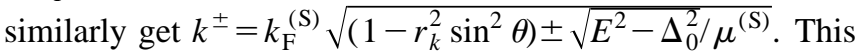
way of including the angle dependence is in accordance with Refs. 6, 8, 11, 12, 17, and 18 but differs from the approach of Chaudhuri and Bagwell ${ }^{7}$ in which the angle corrections to the 1D expressions for $q^{ \pm}$and $k^{ \pm}$are approximated by $\cos \theta$ projection factors. At low temperatures $E / \mu^{(\mathrm{N})}$ $\sim \sqrt{E^{2}-\Delta_{0}^{2}} / \mu^{(\mathrm{S})} \ll 1$, and therefore, we apply the Andreev approximation: $k^{+}=k^{-}=k_{z}^{(\mathrm{S})}$ and $q^{+}=q^{-}=k_{z}^{(\mathrm{N})}$. For values of $\mu^{(\mathrm{N}, \mathrm{S})}$ relevant for normal metals and low-temperature superconductors this approximation is valid for angles of incidence $\theta \leqq \pi / 2$. Semiconductors have much lower Fermi energies as compared to those of normal metals, but, even for $T / T_{\mathrm{F}} \sim 1 / 50$ the approximation is reasonable. For angles in the vicinity of $\theta \sim \pi / 2$ the approximation becomes inaccurate. However, quasiparticles with vanishing perpendicular momentum do not contribute significantly to the perpendicular current and their effect in the IV curves and related quantities will thus not be important for semiconductors either. We have in fact checked this for the excess current by numerically solving Eq. (16) and found less than a half percent deviations for the GaAs two-dimensional electron gas (2DEG) considered in Fig. 2. With these approximations the amplitudes become

$$
a=\frac{u_{0} v_{0}}{\gamma}
$$




$$
\begin{gathered}
b=-\frac{\left(u_{0}^{2}-v_{0}^{2}\right)\left[\Gamma\left(\frac{Z}{\cos \theta}\right)^{2}+\frac{1-\Gamma^{2} r^{2}}{4 \Gamma r}+i \Gamma \sqrt{r} \frac{Z}{\cos \theta}\right]}{\gamma}, \\
c=\frac{u_{0}\left(\frac{1+\Gamma r}{2}-i \Gamma \sqrt{r} \frac{Z}{\cos \theta}\right)}{\gamma}, \\
d=\frac{i v_{0}\left(\Gamma \sqrt{r} \frac{Z}{\cos \theta}-i \frac{1-\Gamma r}{2}\right)}{\gamma},
\end{gathered}
$$

where we define $\gamma \equiv u_{0}^{2}+\left(u_{0}^{2}-v_{0}^{2}\right) Z_{\text {eff }}^{2}(\theta)$, and where

$$
Z_{\mathrm{eff}}(\theta)=\sqrt{\Gamma(\theta)(Z / \cos \theta)^{2}+\left[\Gamma(\theta) r_{v}-1\right]^{2} / 4 \Gamma(\theta) r_{v}}
$$

is an effective barrier strength, $r_{v} \equiv v_{\mathrm{F}}^{(\mathrm{N})} / v_{\mathrm{F}}^{(\mathrm{S})}$ is the Fermi velocity ratio and $\Gamma(\theta) \equiv \cos \theta / \sqrt{1-r_{k}^{2} \sin ^{2} \theta}$. The dimensionless barrier strength $Z \equiv H / \hbar \sqrt{v_{\mathrm{F}}^{(\mathrm{N})} v_{\mathrm{F}}^{(\mathrm{S})}}$ was introduced by Blonder and Tinkham. ${ }^{9}$

In order to obtain the scattering probabilities $A, B, C$, and $D$ we use the conservation of the probability current density, Eq. (3). For the $z$ direction this yields

$$
1=\underbrace{|a|^{2}}_{A}+\underbrace{|b|^{2}}_{B}+\underbrace{\Theta\left(|E|-\Delta_{0}\right) \frac{\left|u_{0}\right|^{2}-\left|v_{0}\right|^{2}}{\Gamma r}|c|^{2}}_{C}+\underbrace{\Theta\left(|E|-\Delta_{0}\right) \frac{\left|u_{0}\right|^{2}-\left|v_{0}\right|^{2}}{\Gamma r}|d|^{2}}_{D} .
$$

It turns out that the scattering probabilities of the BTK model can still be applied provided that the dimensionless barrier strength is replaced by the introduced effective barrier strength given in Eq. (14). For perpendicular incidence ( $\theta$ $=0)$ this result agrees with the BTK result $\left(r_{v}=1\right)$ (Ref. 2) and the Blonder-Tinkham result, ${ }^{9}$ which includes the possibility of a Fermi velocity mismatch. For a general angle of incidence and matching Fermi velocities and Fermi momenta $\left(r_{v}=r_{k}=1\right)$ the result reduces to that obtained by Kupka. ${ }^{6}$

As mentioned, the wave vectors of the transmitted waves have the form $k_{z}^{(\mathrm{S})}=k_{\mathrm{F}}^{(\mathrm{S})} \sqrt{1-r_{k}^{2} \sin ^{2} \theta}$. The square root defines a critical angel of incidence $\theta_{c}$, above which the solutions are evanescent and below which we have propagating waves, i.e., $\theta_{c}=\arcsin \left(1 / r_{k}\right)$ for $r_{k}>1$. The physical reason for the critical angle is that the parallel momentum exceeds the Fermi momentum of the superconductor and thus momentum cannot be conserved. For $r_{k} \leqslant 1$ there is no critical angle due to the parallel momentum not being conserved. Going beyond the Andreev approximation introduces another energy-dependent critical angle $\widetilde{\theta}_{c}$ $=\arcsin \sqrt{1-E / \mu^{(\mathrm{N})}}$ caused by the wave vector $q^{-}$of the Andreev scattering state being imaginary. ${ }^{17}$ However, the later critical angle has little consequences for our results for the same reasons as when we discussed the validity of the Andreev approximation. In Sec. V, we suggest how the angle dependence may be probed.

The directions of the reflected and transmitted waves can be obtained by considering the probability current density and the result is sketched in Fig. 1. The angle of reflection $\theta_{r}$ coincides with the angle of incidence $\theta$ and the angle of transmission is given by

$$
\sin \theta_{t}=r_{k} \sin \theta
$$

in analogy with Snell's law in optics as it was also found by Kupriyanov. ${ }^{10}$ The general results for the scattering prob- abilities including the possibility of a Fermi velocity mismatch are summarized in Table I. We conclude that the scattering probabilities of the BTK model still apply, provided that the dimensionless barrier strength is replaced by an angle-dependent effective barrier strength.

As the angle of incidence is increased, we observe an increasing effective barrier strength and therefore Andreev reflection is suppressed when the parallel momentum becomes much larger than the perpendicular momentum. ${ }^{6}$ In the same way, normal reflection increases when the parallel momentum increases. Nevertheless, we still have unit prob-

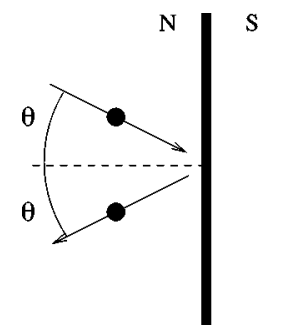

normal reflection

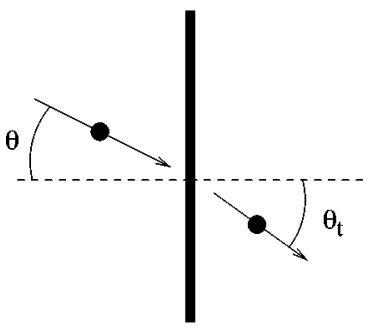

transmission without branch-crossing

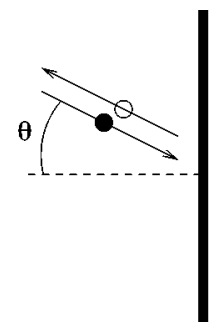

Andreev reflection

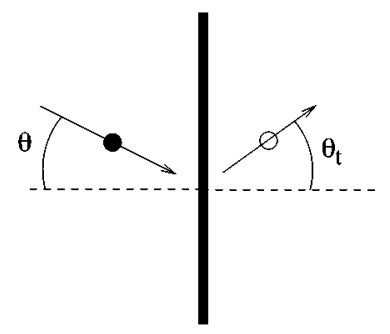

transmission with branch-crossing
FIG. 1. Directions of the transmitted and reflected waves in real space. The full dots $(\mathbf{O})$ represent quasiparticles of predominantly electronlike character and the open dots $(\bigcirc)$ represent quasiparticles of predominantly holelike character. 
TABLE I. Scattering probabilities at NS interface as a function of the normalized excitation energy $\widetilde{E}$ $\equiv E / \Delta_{0}$.

\begin{tabular}{|c|c|c|c|}
\hline & $|\widetilde{E}|<1, \theta<\theta_{c}$ & $|\widetilde{E}|>1, \theta<\theta_{c}$ & $\theta \geqslant \theta_{c}$ \\
\hline \multirow[t]{2}{*}{$A(\widetilde{E}, \theta)$} & 1 & 1 & \multirow[t]{2}{*}{0} \\
\hline & $\widetilde{E}^{2}+\left(1-\widetilde{E}^{2}\right)\left(1+2 Z_{\text {eff }}^{2}(\theta)\right)^{2}$ & $\overline{\left[|\widetilde{E}|+\sqrt{\widetilde{E}^{2}-1}\left(1+2 Z_{\mathrm{eff}}^{2}(\theta)\right)\right]^{2}}$ & \\
\hline \multirow[t]{2}{*}{$B(\widetilde{E}, \theta)$} & \multirow[t]{2}{*}{$1-A(\widetilde{E}, \theta)$} & $4\left(\widetilde{E}^{2}-1\right)\left(Z_{\mathrm{eff}}^{4}(\theta)+Z_{\mathrm{eff}}^{2}(\theta)\right)$ & \multirow[t]{2}{*}{1} \\
\hline & & {$\left[|\widetilde{E}|+\sqrt{\widetilde{E}^{2}-1}\left(1+2 Z_{\mathrm{eff}}^{2}(\theta)\right)\right]^{2}$} & \\
\hline \multirow[t]{2}{*}{$C(\widetilde{E}, \theta)$} & \multirow[t]{2}{*}{0} & $2 \sqrt{\widetilde{E}^{2}-1}\left(|\widetilde{E}|+\sqrt{\widetilde{E}^{2}-1}\right)\left(1+Z_{\text {eff }}^{2}(\theta)\right)$ & \multirow[t]{2}{*}{0} \\
\hline & & {$\left[|\widetilde{E}|+\sqrt{\widetilde{E}^{2}-1}\left(1+2 Z_{\mathrm{eff}}^{2}(\theta)\right)\right]^{2}$} & \\
\hline \multirow[t]{2}{*}{$D(\widetilde{E}, \theta)$} & \multirow[t]{2}{*}{0} & $2 \sqrt{\widetilde{E}^{2}-1}\left(|\widetilde{E}|-\sqrt{\widetilde{E}^{2}-1}\right) Z_{\text {eff }}^{2}(\theta)$ & \multirow[t]{2}{*}{0} \\
\hline & & {$\left[|\widetilde{E}|+\sqrt{\widetilde{E}^{2}-1}\left(1+2 Z_{\mathrm{eff}}^{2}(\theta)\right)\right]^{2}$} & \\
\hline
\end{tabular}

ability for Andreev reflection at the gap edge for all angles of incidence $\left(\theta<\theta_{c}\right)$ and for all barrier strengths.

\section{CURRENT-VOLTAGE CHARACTERISTIC, EXCESS CURRENT, AND DIFFERENTIAL CONDUCTANCE}

We calculate the current on the normal side of the interface where the current is carried only by single quasiparticles and no supercurrent. The current density in the $z$ direction is given by

$$
J_{z}=\sum_{\sigma} \int \frac{d^{d} \mathbf{k}}{(2 \pi)^{d}} e \mathbf{v} \cdot \hat{\mathbf{e}}_{z} f^{(\mathrm{N})}(\mathbf{k}),
$$

where $d=1,2,3$ is the dimension of the electron gas and $f^{(\mathrm{N})}(\mathbf{k})$ is the nonequilibrium distribution function on the normal side of the interface. This approach neglects coherent effects of the propagation of electronlike and holelike quasiparticles in the normal region and it applies to NS interfaces with a ballistic normal region and/or NS interfaces where the length of the normal region is large on the scale of the phase coherence length. The integration is performed using polar coordinates appropriate for a 1D electron gas, a twodimensional (2D) electron gas, and a 3D electron gas, respectively. The 1D case corresponds to the BTK model.

In general the nonequilibrium distribution function can be found from a suitable transport equation, e.g., Boltzmann equation. Instead of taking this path, we follow BTK and assume that all quasiparticles incident from the reservoir are distributed in accordance with the Fermi-Dirac equilibrium distribution function with a shift in energy due to the applied voltage. When current flows, the reservoir is not in true equilibrium. However, the voltage drop across the normal region can be accounted for by an Ohmic series resistance. We calculate $f^{(\mathrm{N})}(E, \theta)=\Theta(\pi / 2-\theta) f_{\rightarrow}^{(\mathrm{N})}(E, \theta)+\Theta(\theta-\pi / 2) f_{\leftarrow}^{(\mathrm{N})}(E, \theta)$ by considering the two subpopulations separately. If we take the chemical potential of the superconductor as reference, we get $f_{\rightarrow}^{(\mathrm{N})}(E, \theta, V)=f_{0}(E-e V)$ for the subpopulation of quasiparticles with a positive momentum in the $z$ direction. The subpopulation of quasiparticles with a negative momentum is

$$
\begin{aligned}
f_{\leftarrow}^{(\mathrm{N})}(E, \pi-\theta, V)= & A(-E, \theta)\left[1-f_{\rightarrow}^{(\mathrm{N})}(-E, \theta, V)\right] \\
& +B(E, \theta) f_{\rightarrow}^{(\mathrm{N})}(E, \theta, V) \\
& +C(E, \theta) f_{\leftarrow}^{(\mathrm{S})}\left(E, \theta_{S}, V\right) \\
& +D(E, \theta) f_{\leftarrow}^{(\mathrm{S})}\left(E, \theta_{S}, V\right) .
\end{aligned}
$$

Here, the first term represents Andreev reflection of timereversed quasiparticles, the second term represents normal reflection, and the last two terms represent transmission of quasiparticles from the superconductor where $f_{\leftarrow}^{(\mathrm{S})}\left(E, \theta_{S}, V\right)$ $=f_{0}(E)$ and $\theta_{S}=\arcsin \left(r_{k} \sin \theta\right)$. Using the translational invariance along the interface, the sum rule $1=A+B+C$ $+D$, and the symmetries with respect to energy yields the normalized current

$$
I=\frac{\Delta_{0}}{e R_{\mathrm{N}}} \int_{-\infty}^{\infty} \mathrm{d} \widetilde{E} \bar{T}(\widetilde{E})\left[f_{0}\left(\widetilde{E}-e V / \Delta_{0}\right)-f_{0}(\widetilde{E})\right]
$$

where $\widetilde{E} \equiv E / \Delta_{0}$ is the normalized excitation energy, $R_{\mathrm{N}}$ is the normal state resistance, and

$$
\begin{gathered}
\bar{T}_{1 \mathrm{D}}(\widetilde{E}) \equiv[1+A(\widetilde{E}, 0)-B(\widetilde{E}, 0)], \\
\bar{T}_{2 \mathrm{D}}(\widetilde{E}) \equiv \int_{0}^{\pi / 2} \mathrm{~d} \theta \frac{\cos \theta}{2 \pi}[1+A(\widetilde{E}, \theta)-B(\widetilde{E}, \theta)], \\
\bar{T}_{3 \mathrm{D}}(\widetilde{E}) \equiv \int_{0}^{\pi / 2} \mathrm{~d} \theta \frac{\sin \theta \cos \theta}{2}[1+A(\widetilde{E}, \theta)-B(\widetilde{E}, \theta)],
\end{gathered}
$$

are effective transmission coefficients for electrical current. The current for two- and three-dimension systems have the same qualitative form as in the 1D BTK model. However, quantitative changes are seen in the excess current and the differential conductance.

The effective transmission coefficients are in general larger than the corresponding normal state transmission coefficients and this effect gives rise to a voltage dependent excess current compared to the normal state, where often the high-voltage limit 

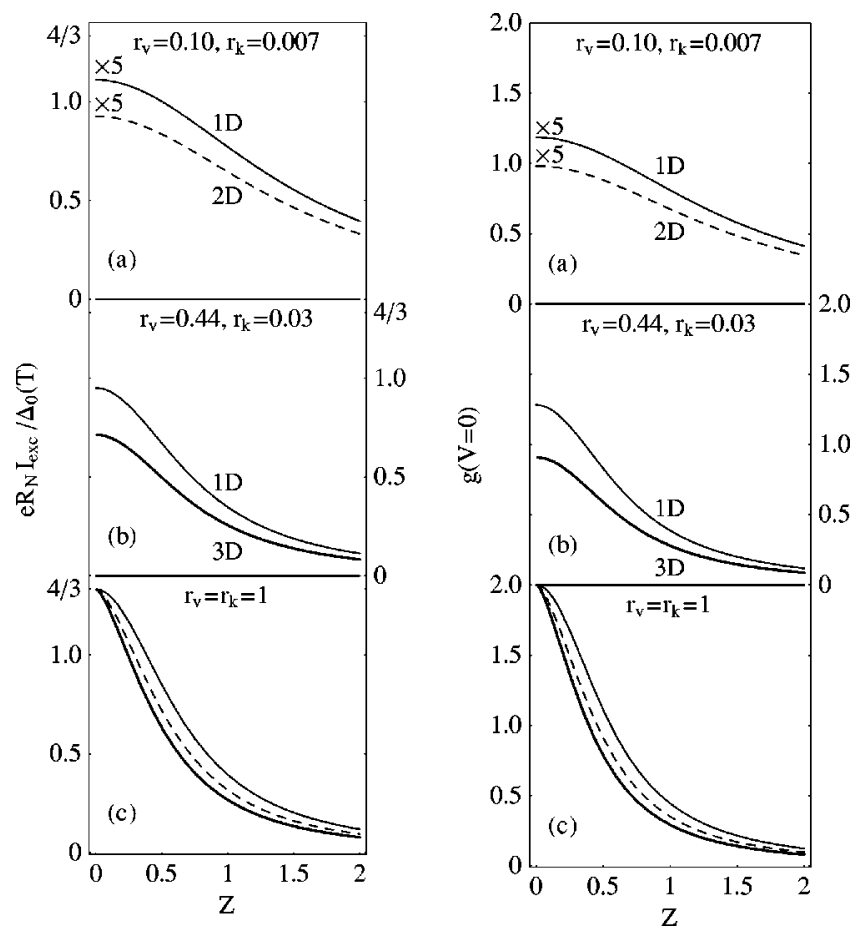

FIG. 2. Left panel: Normalized excess current $e R_{\mathrm{N}} I_{\mathrm{exc}} / \Delta_{0}(T)$ as a function of the dimensionless barrier strength $Z$. Right panel: Normalized differential conductance at zero bias $g(V=0)$ as a function of the dimensionless barrier strength $Z$. The thin lines, dashed lines, and thick lines correspond to the 1D BTK model, the 2D, and 3D calculations, respectively. The temperature dependence of the excess current is entirely contained within the normalization through $\Delta_{0}(T)$ and the differential conductance is plotted at $T$ $=0 \mathrm{~K}$. The excess current and the zero bias differential conductance are shown for (a) parameters appropriate for a 2D GaAs-Al interface with a Fermi temperature $T_{\mathrm{F}} \simeq 100 \mathrm{~K}$ of GaAs, (b) parameters appropriate for the 3D GaAs-Al interface studied by Taboryski et al., ${ }^{19}$ and (c) matching Fermi velocities and Fermi momenta. For the shown $2 \mathrm{D}$ result in (a) and $3 \mathrm{D}$ result in (b) the overestimation of $Z$ for a perfect interface is 0.68 and 0.45 , respectively.

$$
I_{\mathrm{exc}} \equiv \lim _{e V \gg \Delta_{0}}\left[I(V)-\lim _{\Delta_{0} \rightarrow 0} I(V)\right]
$$

is of interest from an experimental point of view.

We have shown above that a large parallel momentum suppresses the Andreev reflection probability and thus we expect to see a lower excess current in the three- or twodimensional limit as compared to the case of perpendicular incidence. This is seen in the left panel of Fig. 2. For perfect $(Z=0)$ 2D and 3D interfaces with nonmatching Fermi velocities and Fermi momenta, the 1D BTK model overestimates the barrier strength $Z$ significantly. For the shown 2D and $3 \mathrm{D}$ results the overestimation of $Z$ for a perfect interface is 0.68 and 0.45 , respectively.

At low temperatures the normalized differential conductance, $g \equiv G_{\mathrm{NS}} / G_{\mathrm{NN}}$, is given by

$$
g(V)=\bar{T}\left(e V / \Delta_{0}\right) / \bar{T}_{\mathrm{N}},
$$

where $\bar{T}_{\mathrm{N}}$ is the effective transmission probability when the superconductor is in the normal state. In the right panel of Fig. 2, results at zero bias are shown. Similarly to the excess

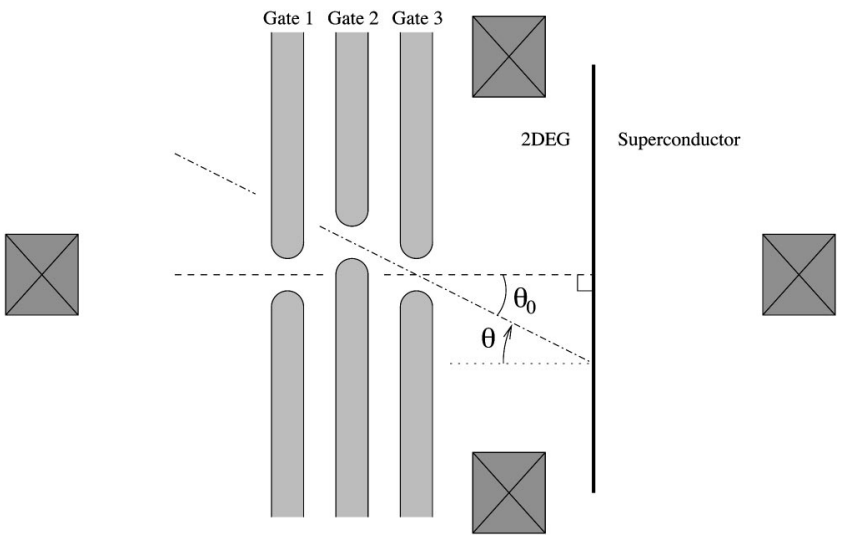

FIG. 3. Interface between a ballistic $2 \mathrm{DEG}$ and a superconductor. The three gates may individually be applied a gate-voltage and in this way it is possible to have perpendicular incidence or a finite angle of incidence defined by for example gate 2 and gate 3 .

current, we find a lower zero-bias conductance with raising dimensionality as compared to the case of transport with perpendicular incidence.

As an application of the present results, we now consider recent experiments by Taboryski et al. ${ }^{19}$ who reported on Andreev reflections at interfaces between GaAs (3DEG) and superconducting $\mathrm{Al}$ films. The material parameters are $r_{v}$ $\simeq 0.44$ and $r_{k} \simeq 0.03$ and from the excess current of the 1D BTK model, Taboryski et al. deduce the dimensionless barrier strength $Z_{\mathrm{fit}}$ to fall in the range from 0.7 to 0.9. Comparing with (b) in the left panel of Fig. 2 we find the barrier strength to fall in the range from 0.5 to 0.7 . For GaAs, the energy dependence of the effective mass due to nonparabolicity is negligible within $\sim 50 \Delta_{\mathrm{Al}}$ of the Fermi level ${ }^{20}$ for both the cases considered in Fig. 2. Since the zero-bias conductance is a Fermi-surface property no restrictions have been made by neglecting the energy dependence of the effective mass. For the high voltage limit of the excess current, the corrections due to a nonparabolic conduction band are small. For InAs, as considered by Schüssler and Kümmel ${ }^{11}$ the energy dependence due to nonparabolicity is more pronounced.

\section{SUGGESTED EXPERIMENT}

Benistant et al. $^{21}$ have studied the angle dependence of Andreev scattering at $\mathrm{Ag}-\mathrm{Pb}$ interfaces experimentally by using a magnetic focusing technique. The quasiparticles are injected to a very clean (ballistic) 3D Ag crystal through a point contact and the angle of incidence at the NS interface is controlled by a weak magnetic field. We suggest a variant based on an interface between a ballistic two-dimensional electron-gas (2DEG) and a superconductor and the technological opportunity of defining the angle of incidence geometrically. By applying gates on top of the 2DEG, it is possible to control the angle of incidence as sketched in Fig. 3. If gate 1 and gate 3 are both negatively biased whereas gate 2 is turned off then quasiparticles have perpendicular incidence. However, biasing gate 2 and gate 3 , while gate 1 is turned off, a finite angle of incidence can be achieved.

The angle dependence can be studied by measuring the differential conductance. At low temperatures the normalized 
differential conductance, $\quad g(e V, \theta) \equiv G_{\mathrm{NS}}(e V, \theta) /$ $G_{\mathrm{NN}}(e V, \theta)$, is given by

$$
g(e V, \theta)=\frac{1+T(\theta) A(e V, \theta)-T(-\theta) B(e V, \theta)}{1-T(-\theta) \widetilde{B}(\theta)},
$$

where $\widetilde{B}=Z_{\text {eff }}^{2} /\left(1+Z_{\text {eff }}^{2}\right)$ is the normal reflection probability when the superconductor is in the normal state. The transmission for quasiparticles leaving the interface through the gates, $T(\theta)$, is peaked around $\theta=\theta_{0}$, with a width depending on the geometry. The possibility of multiple Andreev reflections can be neglected if the phase-relaxation length is less than four times the distance between the interface and the gate nearest to the interface.

The experimental curves for $g(e V, 0)$ and $g\left(e V, \theta_{0}\right)$ may be fitted to Eq. (25) with the transmission, $T\left(\theta_{0}\right)$, and the barrier strength, $Z$, as fitting parameters. Further information on $Z$ and $T\left(\theta_{0}\right)$ may be obtained from the normal state conductance. When the superconductor is in the normal state the Landauer formula yields the conductance

$$
G_{\mathrm{NN}}\left(\theta_{0}\right)=\frac{2 e^{2}}{h} M T\left(\theta_{0}\right)\left[1-\widetilde{B}\left(\theta_{0}\right)\right]
$$

where $M$ is the number of modes. Thus, agreement with the angle dependence is found if fits of the experimental curves for $g(e V, 0)$ and $g\left(e V, \theta_{0}\right)$ can be obtained using the same $Z$ value.

\section{DISCUSSION AND CONCLUSION}

The angle dependence of scattering at NS interfaces is of important consequence when the NS interface has two or three-dimensional nature. The parallel degrees of freedom also have important consequences in superconducting meso- scopic transport. If the current is carried by more than a single mode different modes represent different momenta parallel to the interface, and thus the scattering amplitudes depend on the mode index. More details may be found in a review of scattering theory in mesoscopic NS structures by Beenakker. $^{18}$

We have investigated the angle-dependence of scattering of quasiparticles at NS interfaces using the framework of Bogoliubov-de Gennes. As a main result the scattering probabilities of the BTK model may still be applied provided that the scattering strength is replaced by an effective angledependent barrier strength. This modified effective scattering parameter agrees with previous calculations of BTK, ${ }^{2}$ Blonder and Tinkham, ${ }^{9}$ and Kupka. ${ }^{6}$ One of the consequences is that the Andreev reflection is suppressed for large angles of incidence and the normal reflection is increased towards unity. In the presence of a Fermi momentum mismatch, we find the angles of incidence and transmission to be related and in analogy with Snell's law, we find that above a certain critical angle of incidence we only have normal reflection.

Furthermore, the results of the angle dependence have been applied to NS interfaces with one, two and threedimensional nature where we find that the 1D BTK model overestimates the barrier strength. Calculations show that for certain material parameters and clean interfaces the corrections may be significant. However, the over-all qualitative predictions of the 1D BTK model are found to agree with the new calculations.

\section{ACKNOWLEDGMENT}

We would like to thank J. Bindslev Hansen, J. Kutchinsky, and R. Taboryski for useful discussions.
${ }^{1}$ A.F. Andreev, Zh. Éksp. Teor. Fiz. 46, 1823 (1964) [Sov. Phys. JETP 19, 1228 (1964)].

${ }^{2}$ G.E. Blonder, M. Tinkham, and T.M. Klapwijk, Phys. Rev. B 25, 4515 (1982).

${ }^{3}$ P.G. de Gennes, Superconductivity of Metals and Alloys (Benjamin, New York, 1966).

${ }^{4}$ G.B. Arnold, J. Low Temp. Phys. 59, 143 (1985); 68, 1 (1987).

${ }^{5}$ M. Kupka, J. Phys.: Condens. Matter 2, 10599 (1990); Physica C 221, 346 (1994).

${ }^{6}$ M. Kupka, Physica C 281, 91 (1997).

${ }^{7}$ S. Chaudhuri and P.F. Bagwell, Phys. Rev. B 51, 16936 (1995). [Notice the different notation in Eqs. (A1) and (A2): $q^{ \pm} \leftrightarrow k_{ \pm}^{x}$ and $\left.k^{ \pm} \leftrightarrow q_{ \pm}^{x}.\right]$

${ }^{8}$ H. De Raedt, K. Michielsen, and T.M. Klapwijk, Phys. Rev. B 50, 631 (1994).

${ }^{9}$ G.E. Blonder and M. Tinkham, Phys. Rev. B 27, 112 (1983).

${ }^{10}$ M.Yu. Kupriyanov, Fiz. Nizk. Temp. 7, 700 (1981) [Sov. J. Low Temp. Phys. 7, 342 (1981)].

${ }^{11}$ U. Schüssler and R. Kümmel, Phys. Rev. B 47, 2754 (1993).

${ }^{12}$ A. Chrestin, T. Matsuyama, and U. Merkt, Phys. Rev. B 49, 498 (1994)
${ }^{13}$ T. Akazaki, H. Takayanagi, J. Nitta, and T. Enoki, Appl. Phys. Lett. 68, 418 (1996).

${ }^{14} \mathrm{G}$. Bastard, Wave Mechanics Applied to Semiconductor Heterostructures (Halstead, New York, 1988).

${ }^{15}$ M.G. Burt, Phys. Rev. B 50, 7518 (1994).

${ }^{16}$ S. Gasiorowicz, Quantum Physics (Wiley, New York, 1974).

${ }^{17}$ O. S̆ Sipr and B.L. Györffy, J. Phys.: Condens. Matter 8, 169 (1996); J. Low Temp. Phys. 106, 315 (1997).

${ }^{18}$ C.W.J. Beenakker, in Mesoscopic Quantum Physics, edited by E. Akkermans, G. Montambaux, and J.-L. Pichard (North-Holland, Amsterdam, 1995).

${ }^{19}$ R. Taboryski, T. Clausen, J. Bindslev Hansen, J.L. Skov, J. Kutchinsky, C.B. Sørensen, and P.E. Lindelof, Appl. Phys. Lett. 69, 656 (1996).

${ }^{20}$ S. Adachi, in Properties of Aluminum Gallium Arsenide, edited by S. Adachi, emis datareviews series No. 7 (Inspec, London, 1993).

${ }^{21}$ P.A.M. Benistant, A.P. van Gelder, H. van Kempen, and P. Wyder, Phys. Rev. B 32, 3351 (1985). 